\title{
Note on the cross-section in the semi-tensor bundle
}

\section{Furkan Yildirim}

Narman Vocational Training School, Ataturk University, Erzurum, Turkey

Abstract: Using the fiber bundle M over a manifold B, we define a semi-tensor (pull-back) bundle tB of type (p,q). The complete and horizontal lift of projectable geometric objects on $\mathrm{M}$ to the semi-tensor (pull-back) bundle tB of type (p,q) are presented. The main purpose of this paper is to study the behaviour of complete lift of vector and affinor (tensor of type $(1,1)$ ) fields on cross-sections for pull-back (semi-tensor) bundle tB of type (p,q).

Keywords: Vector field, complete lift, horizontal lift, pull-back bundle, cross-section, semi-tensor bundle.

\section{Introduction}

Let $M_{n}$ be an $n$-dimensional differentiable manifold of class $C^{\infty}$ and $\pi_{1}: M_{n} \rightarrow B_{m}$ the differentiable bundle determined by a submersion $\pi_{1}$. Suppose that $\left(x^{i}\right)=\left(x^{a}, x^{\alpha}\right), a, b, \ldots=1, \ldots, n-m ; \alpha, \beta, \ldots=n-m+1, \ldots, n ; i, j, \ldots=1,2, \ldots, n$ is a system of local coordinates adapted to the bundle $\pi_{1}: M_{n} \rightarrow B_{m}$, where $x^{\alpha}$ are coordinates in $B_{m}$, and $x^{a}$ are fiber coordinates of the bundle $\pi_{1}: M_{n} \rightarrow B_{m}$. If $\left(x^{i^{\prime}}\right)=\left(x^{a^{\prime}}, x^{\alpha^{\prime}}\right)$ is another system of local adapted coordinates in the bundle, then we have

$$
\left\{\begin{array}{l}
x^{a^{\prime}}=x^{a^{\prime}}\left(x^{b}, x^{\beta}\right), \\
x^{\alpha^{\prime}}=x^{\alpha^{\prime}}\left(x^{\beta}\right) .
\end{array}\right.
$$

The Jacobian of (1) has components

$$
\left(A_{j}^{i^{\prime}}\right)=\left(\frac{\partial x^{i^{\prime}}}{\partial x^{j}}\right)=\left(\begin{array}{cc}
A_{b}^{a^{\prime}} & A_{\beta}^{a^{\prime}} \\
0 & A_{\beta}^{\alpha^{\prime}}
\end{array}\right),
$$

where

$$
A_{\beta}^{\alpha^{\prime}}=\frac{\partial x^{\alpha^{\prime}}}{\partial x^{\beta}}
$$

Let $\left(T_{q}^{p}\right)_{x}\left(B_{m}\right)\left(x=\pi_{1}(\widetilde{x}), \widetilde{x}=\left(x^{a}, x^{\alpha}\right) \in M_{n}\right)$ be the tensor space at a point $x \in B_{m}$ with local coordinates $\left(x^{1}, \ldots, x^{m}\right)$, we have the holonomous frame field

$$
\partial_{x^{i_{1}}} \otimes \partial_{x^{i_{2}}} \otimes \ldots \otimes \partial_{x^{i_{p}}} \otimes d x^{j_{1}} \otimes d x^{j_{2}} \otimes \ldots \otimes d x^{j_{q}}
$$

for $i \in\{1, \ldots, m\}^{p}, j \in\{1, \ldots, m\}^{q}$, over $U \subset B_{m}$ of this tensor bundle, and for any $(p, q)$-tensor field $t$ we have [[6], p.163]:

$$
t \mid U=t_{j_{1} \ldots j_{q}}^{i_{1} \ldots i_{p}} \partial_{x_{1}} \otimes \partial_{x_{2}} \otimes \ldots \otimes \partial_{x^{i_{p}}} \otimes d x^{j_{1}} \otimes d x^{j_{2}} \otimes \ldots \otimes d x^{j_{q}}
$$

then by definition the set of all points $\left(x^{I}\right)=\left(x^{a}, x^{\alpha}, x^{\bar{\alpha}}\right), x^{\bar{\alpha}}=t_{j_{1} \ldots j_{q}}^{i_{1} \ldots i_{p}}, \bar{\alpha}=\alpha+m^{p+q}, I, J, \ldots=1, \ldots, n+m^{p+q}$ is a semi-tensor bundle $t_{q}^{p}\left(B_{m}\right)$ over the manifold $M_{n}$. 
The semi-tensor bundle $t_{q}^{p}\left(B_{m}\right)$ has the natural bundle structure over $B_{m}$, its bundle projection $\pi: t_{q}^{p}\left(B_{m}\right) \rightarrow B_{m}$ being defined by $\pi:\left(x^{a}, x^{\alpha}, x^{\bar{\alpha}}\right) \rightarrow\left(x^{\alpha}\right)$. If we introduce a mapping $\pi_{2}: t_{q}^{p}\left(B_{m}\right) \rightarrow M_{n}$ by $\pi_{2}:\left(x^{a}, x^{\alpha}, x^{\bar{\alpha}}\right) \rightarrow\left(x^{a}, x^{\alpha}\right)$, then $t_{q}^{p}\left(B_{m}\right)$ has a bundle structure over $M_{n}$. It is easily verified that $\pi=\pi_{1} \circ \pi_{2}$.

On the other hand, let $\varepsilon=\pi: E \rightarrow B$ denote a fiber bundle with fiber $F$. Given a manifold $B^{\prime}$ and a map $f: B^{\prime} \rightarrow B$, one can construct in a natural way a bundle over $B^{\prime}$ with the same fiber: Consider the subset

$$
f^{*} E=\left\{\left(b^{\prime}, e\right) \in B^{\prime} \times E \mid f\left(b^{\prime}\right)=\pi(e)\right\}
$$

together with the subspace topology from $B^{\prime} \times E$, and denote by $\pi_{1}: f^{*} E \rightarrow B^{\prime}, \pi_{2}: f^{*} E \rightarrow E$ the projections. $f^{*} \varepsilon=\pi_{1}: f^{*} E \rightarrow B^{\prime}$ is a fiber bundle with fiber $F$, called the pull-back bundle of $\varepsilon$ via $f$ [[5],[7],[11],[13]].

From the above definition it follows that the semi-tensor bundle $\left(t_{q}^{p}\left(B_{m}\right), \pi_{2}\right)$ is a pull-back bundle of the tensor bundle over $B_{m}$ by $\pi_{1}$.

In other words, the semi-tensor bundle (induced or pull-back bundle) of the tensor bundle $\left(T_{q}^{p}\left(B_{m}\right), \tilde{\pi}, B_{m}\right)$ is the bundle $\left(t_{q}^{p}\left(B_{m}\right), \pi_{2}, M_{n}\right)$ over $M_{n}$ with a total space

$$
t_{q}^{p}\left(B_{m}\right)=\left\{\left(\left(x^{a}, x^{\alpha}\right), x^{\bar{\alpha}}\right) \in M_{n} \times\left(T_{q}^{p}\right)_{x}\left(B_{m}\right): \pi_{1}\left(x^{a}, x^{\alpha}\right)=\tilde{\pi}\left(x^{\alpha}, x^{\bar{\alpha}}\right)=\left(x^{\alpha}\right)\right\} \subset M_{n} \times\left(T_{q}^{p}\right)_{x}\left(B_{m}\right) .
$$

To a transformation (1) of local coordinates of $M_{n}$, there corresponds on $t_{q}^{p}\left(B_{m}\right)$ the coordinate transformation

$$
\left\{\begin{array}{l}
x^{a^{\prime}}=x^{a^{\prime}}\left(x^{b}, x^{\beta}\right) \\
x^{\alpha^{\prime}}=x^{\alpha^{\prime}}\left(x^{\beta}\right) \\
x^{\bar{\alpha}^{\prime}}=t_{\alpha_{1}^{\prime} \ldots \alpha_{q}^{\prime}}^{\beta_{1}^{\prime} \ldots \beta_{p}^{\prime}}=A_{\alpha_{1} \ldots \alpha_{p}}^{\beta_{1}^{\prime} \ldots \beta_{p}^{\prime}} A_{\alpha_{1}^{\prime} \ldots \alpha_{q}^{\prime}}^{\beta_{1} \ldots \beta_{q}} t_{\beta_{1} \ldots \beta_{q}}^{\alpha_{1} \ldots \alpha_{p}}=A_{(\alpha)}^{\left(\beta^{\prime}\right)} A_{\left(\alpha^{\prime}\right)}^{(\beta)} x^{\bar{\beta}}
\end{array}\right.
$$

The Jacobian of (2) is given by

$$
\bar{A}=\left(A_{J}^{I^{\prime}}\right)=\left(\begin{array}{ccc}
A_{b}^{a^{\prime}} & 0 & 0 \\
0 & A_{\beta}^{\alpha^{\prime}} & 0 \\
0 & t_{(\sigma)}^{(\alpha)} \partial_{\beta} A_{(\alpha)}^{\left(\beta^{\prime}\right)} A_{\left(\alpha^{\prime}\right)}^{(\sigma)} A_{(\alpha)}^{\left(\beta^{\prime}\right)} A_{\left(\alpha^{\prime}\right)}^{(\beta)}
\end{array}\right)
$$

where $I=(a, \alpha, \bar{\alpha}), J=(b, \beta, \bar{\beta}), I, J \ldots=1, \ldots, n+m^{p+q}, t_{(\sigma)}^{(\alpha)}=t_{\sigma_{1} \ldots \sigma_{q}}^{\alpha_{1} \ldots \alpha_{p}}, A_{\beta}^{\alpha^{\prime}}=\frac{\partial x^{\alpha^{\prime}}}{\partial x^{\beta}}$

It is easily verified that the condition $\operatorname{Det} \bar{A} \neq 0$ is equivalent to the condition:

$$
\operatorname{Det}\left(A_{b}^{a^{\prime}}\right) \neq 0, \operatorname{Det}\left(A_{\beta}^{\alpha^{\prime}}\right) \neq 0, \operatorname{Det}\left(A_{(\alpha)}^{\left(\beta^{\prime}\right)} A_{\left(\alpha^{\prime}\right)}^{(\beta)}\right) \neq 0 .
$$

Also, $\operatorname{dim} t_{q}^{p}\left(B_{m}\right)=n+m^{p+q}$. In the special case $n=m, t_{q}^{p}\left(B_{m}\right)$ is a tensor bundle $T_{q}^{p}\left(B_{m}\right)$ [[9], p.118]. In the special case, the semi-tensor bundles $t_{0}^{1}\left(B_{m}\right)(p=1, q=0)$ and $t_{1}^{0}\left(B_{m}\right)(p=0, q=1)$ are semi-tangent and semi-cotangent bundles, respectively.

We note that semi-tangent and semi-cotangent bundle were examined in [[1],[10],[12]] and [[14],[15]], respectively. Also, Fattaev studied the special class of semi-tensor bundle [3]. We denote by $\mathfrak{I}_{q}^{p}\left(t_{q}^{p}\left(\boldsymbol{B}_{m}\right)\right)$ and $\mathfrak{I}_{q}^{p}\left(\boldsymbol{B}_{m}\right)$ the modules 
over $F\left(t_{q}^{p}\left(B_{m}\right)\right)$ and $F\left(B_{m}\right)$ of all tensor fields of type $(p, q)$ on $t_{q}^{p}\left(B_{m}\right)$ and $B_{m}$ respectively, where $F\left(t_{q}^{p}\left(B_{m}\right)\right)$ and $F\left(B_{m}\right)$ denote the rings of real-valued $C^{\infty}$-functions on $t_{q}^{p}\left(B_{m}\right)$ and $B_{m}$, respectively.

\section{Vertical lifts of tensor fields and $\gamma-$ operator}

If $\psi \in t_{q}^{p}\left(B_{m}\right)$, it is regarded, in a natural way, by contraction on $t_{q}^{p}\left(B_{m}\right)$, which we denote by $\imath \psi$. If $\psi$ has the local expression

$$
\psi=\psi_{i_{1} \ldots i_{p}}^{j_{1} \ldots j_{q}} \partial_{j_{1}} \otimes \ldots \otimes \partial_{j_{q}} \otimes d x^{i_{1}} \otimes \ldots \otimes d x^{i_{p}}
$$

in a coordinate neighborhood $U\left(x^{\alpha}\right) \subset B_{m}$, then $\iota \psi=\psi(t)$ has the local expression

$$
\iota \psi=\psi_{i_{1} \ldots i_{p}}^{j_{1} \ldots j_{q}} t_{j_{1} \ldots j_{q}}^{i_{1} \ldots i_{p}}
$$

with respect to the coordinates $\left(x^{a}, x^{\alpha}, x^{\bar{\alpha}}\right)$ in $\pi^{-1}(U)$ [4].

Suppose that $A \in \mathfrak{I}_{q}^{p}\left(B_{m}\right)$. Then there is a unique vector fields ${ }^{v v} A \in \mathfrak{I}_{0}^{1}\left(t_{q}^{p}\left(B_{m}\right)\right)$ such that for $\psi \in t_{q}^{p}\left(B_{m}\right)$ [8]:

$$
{ }^{v v} A(\imath \psi)={ }^{v} A \circ \pi_{2}=\psi(A) \circ \pi_{1} \circ \pi_{2}=\psi(A) \circ \pi={ }^{v v}(\psi(A)),
$$

where ${ }^{v v}(\psi(A))$ is the vertical lift of the function $\psi(A) \in F\left(B_{m}\right)$. We note that the vertical lift ${ }^{v v} f=f \circ \pi$ of the arbitrary function $f \in F\left(B_{m}\right)$ is constant along each fiber of $\pi: t_{q}^{p}\left(B_{m}\right) \rightarrow B_{m}$. If ${ }^{v v} A={ }^{v v} A^{a} \partial_{a}+{ }^{v v} A^{\alpha} \partial_{\alpha}+{ }^{v v} A^{\bar{\alpha}} \partial_{\bar{\alpha}}$, then we have from (4)

$$
{ }^{v v} A={ }^{v v} A^{a} t_{\beta_{1} \ldots \beta_{q}}^{a_{1} \ldots \alpha_{p}} \partial_{a} \psi_{\alpha_{1} \ldots \alpha_{p}}^{\beta_{1} \ldots \beta_{q}}+{ }^{v v} A^{\alpha} t_{\beta_{1} \ldots \beta_{q}}^{\alpha_{1} \ldots \alpha_{p}} \partial_{\alpha} \psi_{\alpha_{1} \ldots \alpha_{p}}^{\beta_{1} \ldots \beta_{q}}+{ }^{v v} A^{\bar{\alpha}} \psi_{\sigma_{1} \ldots \sigma_{p}}^{\theta_{1} \ldots \theta_{q}}=\psi_{\sigma_{1} \ldots \sigma_{p}}^{\theta_{1} \ldots \theta_{q}} A_{\theta_{1} \ldots \theta_{q}}^{\sigma_{1} \ldots \sigma_{p}} .
$$

But $\psi_{\sigma_{1} \ldots \sigma_{p}}^{\theta_{1} \ldots \theta_{q}}, \partial_{a} \psi_{\alpha_{1} \ldots \alpha_{p}}^{\beta_{1} \ldots \beta_{q}}$ and $\partial_{\alpha} \psi_{\alpha_{1} \ldots \alpha_{p}}^{\beta_{1} \ldots \beta_{q}}$ can take any preassigned valued at each point. Thus we have

$$
{ }^{v v} A^{a} t_{\beta_{1} \ldots \beta_{q}}^{\alpha_{1} \ldots \alpha_{p}}=0,{ }^{v v} A^{\alpha} t_{\beta_{1} \ldots \beta_{q}}^{\alpha_{1} \ldots \alpha_{p}}=0,{ }^{v v} A^{\bar{\alpha}}=A_{\theta_{1} \ldots \theta_{q}}^{\sigma_{1} \ldots \sigma_{p}} .
$$

Hence ${ }^{v v} A^{\alpha}=0$ at all points of $t_{q}^{p}\left(B_{m}\right)$ except possibly those at which all the components $x^{\bar{\alpha}}=t_{\beta_{1} \ldots \beta_{q}}^{\alpha_{1} \ldots \alpha_{p}}$ are zero: that is, at points of the base space. Thus we see that the components ${ }^{v v} A^{\alpha}$ are zero a point such that $x^{\bar{\alpha}} \neq 0$, that is, on $t_{q}^{p}\left(B_{m}\right)-B_{m}$. However, $t_{q}^{p}\left(B_{m}\right)-B_{m}$ is dense in $t_{q}^{p}\left(B_{m}\right)$ and the components of ${ }^{v v} A$ are continuous at every point of $t_{q}^{p}\left(B_{m}\right)$. Hence, we have ${ }^{v v} A^{\alpha}=0$ at all points of $t_{q}^{p}\left(B_{m}\right)$. Consequently, the vertical lift ${ }^{v v} A$ of $A$ to $t_{q}^{p}\left(B_{m}\right)$ has components

$$
{ }^{v v} A=\left(\begin{array}{c}
{ }^{v v} A^{a} \\
{ }^{v v} A^{\alpha} \\
{ }^{v v} A^{\bar{\alpha}}
\end{array}\right)=\left(\begin{array}{l}
0 \\
0 \\
A_{\beta_{1} \ldots \beta_{q}}^{\alpha_{1} \ldots \alpha_{p}}
\end{array}\right),
$$

with respect to the coordinates $\left(x^{a}, x^{\alpha}, x^{\bar{\alpha}}\right)$ on $t_{q}^{p}\left(B_{m}\right)$.

Let $\varphi \in \mathfrak{I}_{1}^{1}\left(B_{m}\right)$. We define a vector field $\gamma \varphi$ in $\pi^{-1}(U)$ by

$$
\left\{\begin{array}{l}
\gamma \varphi=\left(\sum_{\lambda=1}^{p} t_{\beta_{1} \ldots \beta_{q} \ldots \alpha_{p}}^{\alpha_{1}} \varphi_{\varepsilon}^{\alpha_{\lambda}}\right) \frac{\partial}{\partial x^{\bar{\beta}}}, \quad(p \geq 1, q \geq 0) \\
\widetilde{\gamma} \varphi=\left(\sum_{\mu=1}^{q} t_{\beta_{1} \ldots \alpha_{p} \ldots \beta_{q}} \varphi_{\beta_{\mu}}^{\varepsilon}\right) \frac{\partial}{\partial x^{\beta} \bar{\beta}}, \quad(p \geq 0, q \geq 1)
\end{array}\right.
$$

with respect to the coordinates $\left(x^{b}, x^{\beta}, x^{\bar{\beta}}\right)$ on $t_{q}^{p}\left(B_{m}\right)$. From (3) we easily see that the vector fields $\gamma \varphi$ and $\widetilde{\gamma} \varphi$ defined in each $\pi^{-1}(U) \subset t_{q}^{p}\left(B_{m}\right)$ determine respectively global vertical vector fields on $t_{q}^{p}\left(B_{m}\right)$. We call $\gamma \varphi$ (or $\tilde{\gamma} \varphi$ ) the verticalvector lift of the tensor field $\varphi \in \mathfrak{I}_{1}^{1}\left(B_{m}\right)$ to $t_{q}^{p}\left(B_{m}\right)$. For any $\varphi \in \mathfrak{I}_{1}^{1}\left(B_{m}\right)$, if we take account of (3) and (6), we can prove 
that $(\gamma \varphi)^{\prime}=\bar{A}(\gamma \varphi)$ where $\gamma \varphi$ is a vector field defined by

$$
\gamma \varphi=(\gamma \varphi)^{I}=\left(\begin{array}{l}
0 \\
0 \\
\sum_{\lambda=1}^{p} t_{\beta_{1} \ldots \beta_{q}}^{\alpha_{1} \ldots \varepsilon \ldots \alpha_{p}} \varphi_{\varepsilon}^{\alpha_{\lambda}}
\end{array}\right) .
$$

Let $\varphi \in \mathfrak{I}_{1}^{1}\left(B_{m}\right)$. On putting

$$
\tilde{\gamma} \varphi=(\widetilde{\gamma} \varphi)^{I}=\left(\begin{array}{l}
0 \\
0 \\
\sum_{\mu=1}^{q} t_{\beta_{1} \ldots \varepsilon \ldots \beta_{q}}^{\alpha_{1} \ldots \alpha_{p}} \\
\phi_{\beta_{\mu}}^{\varepsilon}
\end{array}\right),
$$

we easily see that $(\widetilde{\gamma} \varphi)^{\prime}=\bar{A}(\widetilde{\gamma} \varphi)$.

\section{Complete lifts of vector fields}

We now denote by $\mathfrak{I}_{q}^{p}\left(M_{n}\right)$ the module over $F\left(M_{n}\right)$ of all tensor fields of type $(p, q)$ on $M_{n}$, where $F\left(M_{n}\right)$ denotes the ring of real-valued $C^{\infty}$-functions on $M_{n}$. Let $\widetilde{X} \in \mathfrak{I}_{0}^{1}\left(M_{n}\right)$ be a projectable vector field [9] with projection $X=X^{\alpha}\left(x^{\alpha}\right) \partial_{\alpha}$ i.e. $\widetilde{X}=\widetilde{X}^{a}\left(x^{a}, x^{\alpha}\right) \partial_{a}+X^{\alpha}\left(x^{\alpha}\right) \partial_{\alpha}$. On putting

$$
{ }^{c c} \widetilde{X}=\left(\begin{array}{l}
{ }^{c} \widetilde{X}^{b} \\
{ }^{c} \widetilde{X}^{\beta} \\
{ }^{c c} \widetilde{X}^{\beta}
\end{array}\right)=\left(\begin{array}{l}
\widetilde{X}^{b} \\
\widetilde{X}^{\beta} \\
\sum_{\lambda=1}^{p} t_{\beta_{1} \ldots \beta_{q}}^{\alpha_{1} \ldots \alpha_{p}} \partial_{\varepsilon} X^{\alpha_{\lambda}}-\sum_{\mu=1}^{q} t_{\beta_{1} \ldots \varepsilon \ldots \beta_{q}}^{\alpha_{1} \ldots \alpha_{p}} \partial_{\beta_{\mu}} X^{\varepsilon}
\end{array}\right),
$$

we easily see that ${ }^{c c} \widetilde{X}^{\prime}=\bar{A}\left({ }^{c c} \widetilde{X}\right)$. The vector field ${ }^{c c} \widetilde{X}$ is called the complete lift of $\widetilde{X}$ to the semi-tensor bundle $t_{q}^{p}\left(B_{m}\right)$.

\section{Horizontal lifts of vector fields}

Let $\widetilde{X} \in \mathfrak{I}_{0}^{1}\left(M_{n}\right)$ be a projectable vector field [9] with projection $X=X^{\alpha}\left(x^{\alpha}\right) \partial_{\alpha}$ i.e. $\widetilde{X}=\widetilde{X}^{a}\left(x^{a}, x^{\alpha}\right) \partial_{a}+X^{\alpha}\left(x^{\alpha}\right) \partial_{\alpha}$. If we take account of (3), we can prove that ${ }^{H H} \widetilde{X}^{\prime}=\bar{A}\left({ }^{H H} \widetilde{X}\right)$, where ${ }^{H H} \widetilde{X}$ is a vector field defined by

$$
{ }^{H H} \widetilde{X}=\left(\begin{array}{l}
\widetilde{X}^{b} \\
\widetilde{X}^{\beta} \\
X^{l}\left(\sum_{\mu=1}^{q} \Gamma_{l \beta_{\mu}}^{\varepsilon} t_{\beta_{1} \ldots \varepsilon \ldots \beta_{q}}^{\alpha_{1} \ldots \alpha_{p}}-\sum_{\lambda=1}^{p} \Gamma_{l \varepsilon}^{\alpha_{\lambda}} t_{\beta_{1} \ldots \beta_{q} \ldots \alpha_{p}}^{\alpha_{1} \ldots \varepsilon}\right)
\end{array}\right),
$$

with respect to the coordinates $\left(x^{b}, x^{\beta}, x^{\bar{\beta}}\right)$ on $t_{q}^{p}\left(B_{m}\right)$. We call ${ }^{H H} \widetilde{X}$ the horizontal lift of the vector field $\widetilde{X}$ to $t_{q}^{p}\left(B_{m}\right)$.

Theorem 1. If $\widetilde{X} \in \mathfrak{I}_{0}^{1}\left(M_{n}\right)$ then

$$
{ }^{c c} \widetilde{X}-{ }^{H H} \widetilde{X}=\gamma(\hat{\nabla} \widetilde{X})-\widetilde{\gamma}(\hat{\nabla} \widetilde{X})
$$

where the symmetric affine connection $\hat{\nabla}$ is the given by $\widehat{\Gamma}_{\beta \theta}^{\alpha}=\Gamma_{\theta \beta}^{\alpha}$. 
Proof. From (7), (8), (9) and (10), we have

$$
\begin{aligned}
& { }^{c c} \widetilde{X}-{ }^{H H} \widetilde{X}=\left(\begin{array}{l}
\widetilde{X}^{b} \\
\widetilde{X}^{\beta} \\
\sum_{\lambda=1}^{p} t_{\beta_{1} \ldots \beta_{q}}^{\alpha_{1} \ldots \alpha_{p}} \partial_{\varepsilon} X^{\alpha_{\lambda}}-\sum_{\mu=1}^{q} t_{\beta_{1} \ldots \varepsilon \ldots \beta_{q}}^{\alpha_{1} \ldots \alpha_{p}} \partial_{\beta_{\mu}} X^{\varepsilon}
\end{array}\right)-\left(\begin{array}{l}
\widetilde{X}^{b} \\
\widetilde{X}^{\beta} \\
X^{l}\left(\sum_{\mu=1}^{q} \Gamma_{l \beta_{\mu}}^{\varepsilon} t_{\beta_{1} \ldots \varepsilon \ldots \beta_{q}}^{\alpha_{1} \ldots \alpha_{p}}-\sum_{\lambda=1}^{p} \Gamma_{l \varepsilon}^{\alpha_{\lambda}} t_{\beta_{1} \ldots \beta_{q}}^{\alpha_{1} \ldots \varepsilon \ldots \alpha_{p}}\right)
\end{array}\right) \\
& =\left(\begin{array}{l}
0 \\
0 \\
\sum_{\lambda=1}^{p} t_{\beta_{1} \ldots \beta_{q}}^{\alpha_{1} \ldots \alpha_{p}}\left(\partial_{\varepsilon} X^{\alpha_{\lambda}}+\Gamma_{l \varepsilon}^{\alpha_{\lambda}} X^{l}\right)-\sum_{\mu=1}^{q} t_{\beta_{1} \ldots \varepsilon \ldots \beta_{q}}^{\alpha_{1} \ldots \alpha_{p}}\left(\partial_{\beta_{\mu}} X^{\varepsilon}+\Gamma_{l \beta_{\mu}}^{\varepsilon} X^{l}\right)
\end{array}\right) \\
& =\left(\begin{array}{l}
0 \\
0 \\
\sum_{\lambda=1}^{p} t_{\beta_{1} \ldots \beta_{q}}^{\alpha_{1} \ldots \varepsilon \alpha_{p}}\left(\partial_{\varepsilon} X^{\alpha_{\lambda}}+\Gamma_{l \varepsilon}^{\alpha_{\lambda}} X^{l}\right)
\end{array}\right)-\left(\begin{array}{l}
0 \\
0 \\
\sum_{\mu=1}^{q} t_{\beta_{1} \ldots \ldots \beta_{q}}^{\alpha_{1} \ldots \alpha_{p}}\left(\partial_{\beta_{\mu}} X^{\varepsilon}+\Gamma_{l \beta_{\mu}}^{\varepsilon} X^{l}\right)
\end{array}\right) \\
& =\left(\begin{array}{l}
0 \\
0 \\
\sum_{\lambda=1}^{p} t_{\beta_{1} \ldots \beta_{q}}^{\alpha_{1} \ldots \alpha_{p}}(\underbrace{\partial_{\varepsilon} X^{\alpha_{\lambda}}+\widehat{\Gamma}_{\varepsilon l}^{\alpha_{\lambda}} X^{l}}_{\hat{\nabla}_{\varepsilon} \widetilde{X}^{\alpha_{\lambda}}})
\end{array}\right)-\left(\begin{array}{l}
0 \\
0 \\
\sum_{\mu=1}^{q} t_{\beta_{1} \ldots \beta_{p} \ldots \beta_{q}}^{\alpha_{1} \ldots \alpha_{p}} \underbrace{\partial_{\beta_{\mu}} X^{\varepsilon}+\widehat{\Gamma}_{\beta_{\mu} l}^{\varepsilon} X^{l}}_{\hat{\nabla}_{\beta_{\mu}} \widetilde{X}^{\varepsilon}})
\end{array}\right)
\end{aligned}
$$

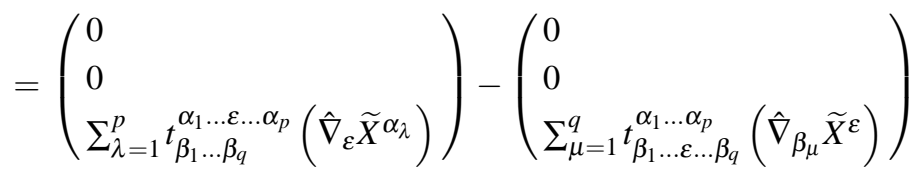

$$
\begin{aligned}
& =\gamma\left(\hat{\nabla}_{\varepsilon} \widetilde{X}^{\alpha_{\lambda}}\right)-\widetilde{\gamma}\left(\hat{\nabla}_{\beta_{\mu}} \widetilde{X}^{\varepsilon}\right)=\gamma(\hat{\nabla} \widetilde{X})-\widetilde{\gamma}(\hat{\nabla} \widetilde{X}) .
\end{aligned}
$$

Thus, we have Theorem 1.

\section{Cross-sections in the semi-tensor bundle}

Let $\xi \in \mathfrak{I}_{q}^{p}\left(B_{m}\right)$ be a tensor field on $B_{m}$. Then the correspondence $x \rightarrow \xi_{x}, \xi_{x}$ being the value of $\xi$ at $x \in M_{n}$, determines a cross-section $\beta_{\xi}$ of semi-tensor bundle. Thus if $\sigma_{\xi}: B_{m} \rightarrow T_{q}^{p}\left(B_{m}\right)$ is a cross-section of $\left(T_{q}^{p}\left(B_{m}\right), \tilde{\pi}, B_{m}\right)$, such that $\tilde{\pi} \circ \sigma_{\xi}=I_{\left(B_{m}\right)}$, an associated cross-section $\beta_{\xi}: M_{n} \rightarrow t_{q}^{p}\left(B_{m}\right)$ of semi-tensor bundle $\left(t_{q}^{p}\left(B_{m}\right), \pi_{2}, M_{n}\right)$ defined by [[2], p. 217-218], [[9], p. 126-127]:

$$
\beta_{\xi}\left(x^{a}, x^{\alpha}\right)=\left(x^{a}, x^{\alpha}, \sigma_{\xi} \circ \pi_{1}\left(x^{a}, x^{\alpha}\right)\right)=\left(x^{a}, x^{\alpha}, \sigma_{\xi}\left(x^{\alpha}\right)\right)=\left(x^{a}, x^{\alpha}, \xi_{\beta_{1} \ldots \beta_{q}}^{\alpha_{1} \ldots \alpha_{p}}\left(x^{\beta}\right)\right) .
$$

If the tensor field $\xi$ has the local components $\xi_{\beta_{1} \ldots \beta_{q}}^{\alpha_{1} \ldots \alpha_{p}}\left(x^{\beta}\right)$, the cross-section $\beta_{\xi}\left(M_{n}\right)$ of $t_{q}^{p}\left(B_{m}\right)$ is locally expressed by

$$
\left\{\begin{array}{l}
x^{b}=x^{b} \\
x^{\beta}=x^{\beta} \\
x^{\bar{\beta}}=\xi_{\beta_{1} \ldots \beta_{q}}^{\alpha_{1} \ldots \alpha_{p}}\left(x^{\beta}\right)
\end{array}\right.
$$

with respect to the coordinates $x^{B}=\left(x^{b}, x^{\beta}, x^{\bar{\beta}}\right)$ in $t_{q}^{p}\left(B_{m}\right)$. Differentiating (11) by $x^{c}$, we see that $n-m$ tangent vector fields $B_{(c)}(c=1, \ldots, n-m)$ to $\beta_{\xi}\left(M_{n}\right)$ have components

$$
B_{(c)}=\frac{\partial x^{B}}{\partial x^{c}}=\partial_{c} x^{B}=\left(\begin{array}{l}
\partial_{c} x^{b} \\
\partial_{c} x^{\beta} \\
\partial_{c} \xi_{\beta_{1} \ldots \beta_{q}}^{\alpha_{1} \ldots \alpha_{p}}
\end{array}\right),
$$


which are tangent to the cross-section $\beta_{\xi}\left(M_{n}\right)$.

Thus $B_{(c)}$ have components

$$
B_{(c)}:\left(B_{(c)}^{B}\right)=\left(\begin{array}{l}
\delta_{c}^{b} \\
0 \\
0
\end{array}\right)
$$

with respect to the coordinates $\left(x^{b}, x^{\beta}, x^{\bar{\beta}}\right)$ in $t_{q}^{p}\left(B_{m}\right)$. Where

$$
\delta_{c}^{b}=A_{c}^{b}=\frac{\partial x^{b}}{\partial x^{c}}
$$

Let $\widetilde{X} \in \mathfrak{I}_{0}^{1}\left(M_{n}\right)$ be a projectable vector field [9] with projection $X=X^{\alpha}\left(x^{\alpha}\right) \partial_{\alpha}$ i.e. $\widetilde{X}=\widetilde{X}^{a}\left(x^{a}, x^{\alpha}\right) \partial_{a}+X^{\alpha}\left(x^{\alpha}\right) \partial_{\alpha}$. We denote by $B X$ the vector field with local components

$$
B X:\left(B_{(c)}^{B} \widetilde{X}^{c}\right)=\left(\begin{array}{l}
\delta_{c}^{b} \widetilde{X}^{c} \\
0 \\
0
\end{array}\right)=\left(\begin{array}{l}
A_{c}^{b} \widetilde{X}^{c} \\
0 \\
0
\end{array}\right)=\left(\begin{array}{l}
\widetilde{X}^{b} \\
0 \\
0
\end{array}\right)
$$

with respect to the coordinates $\left(x^{b}, x^{\beta}, x^{\bar{\beta}}\right)$ in $t_{q}^{p}\left(B_{m}\right)$, which is defined globally along $\beta_{\xi}\left(M_{n}\right)$.

Differentiating (11) by $x^{\theta}$, we have vector fields $C_{(\theta)}(\theta=n-m+1, \ldots, n)$ with components

$$
C_{(\theta)}=\frac{\partial x^{B}}{\partial x^{\theta}}=\partial_{\theta} x^{B}=\left(\begin{array}{l}
\partial_{\theta} x^{b} \\
\partial_{\theta} x^{\beta} \\
\partial_{\theta} \xi_{\beta_{1} \ldots \beta_{q}}^{\alpha_{1} \ldots \alpha_{p}}
\end{array}\right)
$$

which are tangent to the cross-section $\beta_{\xi}\left(M_{n}\right)$.

Thus $C_{(\theta)}$ have components

$$
C_{(\theta)}:\left(C_{(\theta)}^{B}\right)=\left(\begin{array}{l}
A_{\theta}^{b} \\
\delta_{\theta}^{\beta} \\
\partial_{\theta} \xi_{\beta_{1} \ldots \beta_{q}}^{\alpha_{1} \ldots \alpha_{p}}
\end{array}\right)
$$

with respect to the coordinates $\left(x^{b}, x^{\beta}, x^{\bar{\beta}}\right)$ in $t_{q}^{p}\left(B_{m}\right)$. Where

$$
A_{\theta}^{b}=\frac{\partial x^{b}}{\partial x^{\theta}}, \delta_{\theta}^{\beta}=A_{\theta}^{\beta}=\frac{\partial x^{\beta}}{\partial x^{\theta}} .
$$

Let $\widetilde{X} \in \mathfrak{I}_{0}^{1}\left(M_{n}\right)$ be a projectable vector field [9] with projection $X=X^{\alpha}\left(x^{\alpha}\right) \partial_{\alpha}$ i.e. $\tilde{X}=\widetilde{X}^{a}\left(x^{a}, x^{\alpha}\right) \partial_{a}+X^{\alpha}\left(x^{\alpha}\right) \partial_{\alpha}$. We denote by $C X$ the vector field with local components

$$
C X:\left(C_{(\theta)}^{B} X^{\theta}\right)=\left(\begin{array}{l}
A_{\theta}^{b} X^{\theta} \\
X^{\beta} \\
X^{\theta} \partial_{\theta} \xi_{\beta_{1} \ldots \beta_{q}}^{\alpha_{1} \ldots \alpha_{p}}
\end{array}\right)
$$

with respect to the coordinates $\left(x^{b}, x^{\beta}, x^{\bar{\beta}}\right)$ in $t_{q}^{p}\left(B_{m}\right)$, which is defined globally along $\beta_{\xi}\left(M_{n}\right)$. 
On the other hand, the fibre is locally expressed by

$$
\left\{\begin{array}{l}
x^{b}=\text { const. } \\
x^{\beta}=\text { const. } \\
x^{\bar{\beta}}=t_{\beta_{1} \ldots \beta_{q}}^{\alpha_{1} \ldots \alpha_{p}}=t_{\beta_{1} \ldots \beta_{q}}^{\alpha_{1} \ldots \alpha_{p}}
\end{array}\right.
$$

$t_{\beta_{1} \ldots \beta_{q}}^{\alpha_{1} \ldots \alpha_{p}}$ being considered as parameters. Thus, on differentiating with respect to $x^{\bar{\beta}}=t_{\beta_{1} \ldots \beta_{q}}^{\alpha_{1} \ldots \alpha_{p}}$, we easily see that the vector fields $E_{(\bar{\theta})}\left(\bar{\theta}=n+1, \ldots, n+m^{p+q}\right)$ with components

$$
E_{(\bar{\theta})}:\left(E_{(\bar{\theta})}^{B}\right)=\partial_{\bar{\theta}} x^{B}=\left(\begin{array}{l}
\partial_{\bar{\theta}} x^{b} \\
\partial_{\bar{\theta}} x^{\beta} \\
\partial_{\bar{\theta}} t_{\beta_{1} \ldots \beta_{q}}^{\alpha_{1} \ldots \alpha_{p}}
\end{array}\right)=\left(\begin{array}{l}
0 \\
0 \\
\delta_{\beta_{1}}^{\theta_{1}} \ldots \delta_{\beta_{q}}^{\theta_{q}} \delta_{\gamma_{1}}^{\alpha_{1}} \ldots \delta_{\gamma_{p}}^{\alpha_{p}}
\end{array}\right)
$$

is tangent to the fibre, where $\delta$ is the Kronecker symbol.

Let $\xi$ be a tensor field of type $(p, q)$ with local components

$$
\xi=\xi_{\theta_{1} \ldots \theta_{q}}^{\gamma_{1} \ldots \gamma_{p}} d x^{\theta_{1}} \otimes \ldots \otimes d x^{\theta_{q}} \otimes \partial_{\gamma_{1}} \otimes \ldots \otimes \partial_{\gamma_{p}}
$$

on $B_{m}$.

We denote by $E \xi$ the vector field with local components

$$
E \xi:\left(E_{(\bar{\theta})}^{B} \xi_{\theta_{1} \ldots \theta_{q}}^{\gamma_{1} \ldots \gamma_{p}}\right)=\left(\begin{array}{l}
0 \\
0 \\
\xi_{\beta_{1} \ldots \beta_{q}}^{\alpha_{1} \ldots \alpha_{p}}
\end{array}\right)
$$

which is tangent to the fibre.

According to (12) and (13), we define new projectable vector field $H \widetilde{X}$ by

$$
B X+C X=H \widetilde{X}
$$

with respect to the coordinates $\left(x^{b}, x^{\beta}, x^{\bar{\beta}}\right)$ in $t_{q}^{p}\left(B_{m}\right)$, where

$$
H \widetilde{X}=\left(\begin{array}{l}
A_{c}^{b} \widetilde{X}^{c} \\
0 \\
0
\end{array}\right)+\left(\begin{array}{l}
A_{\theta}^{b} X^{\theta} \\
X^{\beta} \\
X^{\theta} \partial_{\theta} \xi_{\beta_{1} \ldots \beta_{q} \ldots \alpha_{p}}
\end{array}\right)=\left(\begin{array}{l}
\widetilde{X}^{b} \\
X^{\beta} \\
X^{\theta} \partial_{\theta} \xi_{\beta_{1} \ldots \beta_{q} \ldots \alpha_{p}}
\end{array}\right) .
$$

We consider in $\pi^{-1}(U) \subset t_{q}^{p}\left(B_{m}\right), n+m^{p+q}$ local vector fields $B_{(c)}, C_{(\theta)}$ and $E_{(\bar{\theta})}$ along $\beta_{\xi}\left(M_{n}\right)$. They form a local family of frames $\left\{B_{(c)}, C_{(\theta)}, E_{(\bar{\theta})}\right\}$ along $\beta_{\xi}\left(M_{n}\right)$, which is called the adapted $(B, C, E)$-frame of $\beta_{\xi}\left(M_{n}\right)$ in $\pi^{-1}(U)$.

We can state following theorem:

Theorem 2. Let $\widetilde{X}$ be a vector field on $M_{n}$ with projection $X$ on $B_{m}$. We have along $\beta_{\xi}\left(M_{n}\right)$ the formulas

(i) ${ }^{c} \widetilde{X}=H \widetilde{X}+E\left(-L_{X} \xi\right)$,

(ii) ${ }^{v v} \xi=E \xi$ 
for any $t \in \mathfrak{I}_{q}^{p}\left(B_{m}\right)$, where $L_{X} \xi$ denotes the Lie derivative of $\xi$ with respect to $X$.

Proof. (i) Using (9), (14) and (15), we have

$$
\begin{aligned}
H \widetilde{X}+E\left(-L_{X} \xi\right) & =\left(\begin{array}{l}
\widetilde{X}^{b} \\
X^{\beta} \\
X^{\theta} \partial_{\theta} \xi_{\beta_{1} \ldots \beta_{q}}^{\alpha_{1} \ldots \alpha_{p}}
\end{array}\right)+\left(\begin{array}{l}
0 \\
0 \\
-X^{\theta} \partial_{\theta} \xi_{\beta_{1} \ldots \beta_{q}}^{\alpha_{1} \ldots \alpha_{p}}-\sum_{\mu=1}^{q} \partial_{\beta_{\mu}} X^{\beta} \xi_{\beta_{1} \ldots \beta \ldots \beta_{q}}^{\alpha_{1} \ldots \alpha_{p}}+\sum_{\lambda=1}^{p} \partial_{\beta} X^{\alpha_{\lambda}} \xi_{\beta_{1} \ldots \beta_{q}}^{\alpha_{1} \ldots \alpha_{p}}
\end{array}\right) \\
& =\left(\begin{array}{l}
\widetilde{X}^{b} \\
X^{\beta} \\
\sum_{\lambda=1}^{p} \partial_{\beta} X^{\alpha_{\lambda}} \xi_{\beta_{1} \ldots \beta_{q}}^{\alpha_{1} \ldots \varepsilon \alpha_{p}}-\sum_{\mu=1}^{q} \partial_{\beta_{\mu}} X^{\beta} \xi_{\beta_{1} \ldots \beta \ldots \beta_{q}}^{\alpha_{1} \ldots \alpha_{p}}
\end{array}\right)={ }^{c c} \widetilde{X} .
\end{aligned}
$$

Thus, we have (16).

(ii) This immediately follows from (5).

On the other hand, on putting $C_{(\bar{\beta})}=E_{(\bar{\beta})}$, we write the adapted frame of $\beta_{\xi}\left(M_{n}\right)$ as $\left\{B_{(b)}, C_{(\beta)}, C_{(\bar{\beta})}\right\}$. The adapted frame $\left\{B_{(b)}, C_{(\beta)}, C_{(\bar{\beta})}\right\}$ of $\beta_{\xi}\left(M_{n}\right)$ is given by the matrix

$$
\widetilde{A}=\left(\widetilde{A}_{B}^{A}\right)=\left(\begin{array}{ccc}
\delta_{b}^{a} & \partial_{\beta} x^{a} & 0 \\
0 & \delta_{\beta}^{\alpha} & 0 \\
0 & \partial_{\beta} \xi_{\alpha_{1} \ldots \alpha_{q}}^{\sigma_{1} \ldots \sigma_{p}} & \delta_{\alpha_{1}}^{\beta_{1}} \ldots \delta_{\alpha_{q}}^{\beta_{q}} \delta_{\gamma_{1}}^{\sigma_{1}} \ldots \delta_{\gamma_{p}}^{\sigma_{p}}
\end{array}\right)
$$

Since the matrix $\widetilde{A}$ in (16) is non-singular, it has the inverse. Denoting this inverse by $(\widetilde{A})^{-1}$, we have

$$
(\widetilde{A})^{-1}=\left(\widetilde{A}_{C}^{B}\right)^{-1}=\left(\begin{array}{ccc}
\delta_{c}^{b} & -\partial_{\theta} x^{b} & 0 \\
0 & \delta_{\theta}^{\beta} & 0 \\
0 & -\partial_{\theta} \xi_{\beta_{1} \ldots \beta_{q}}^{\sigma_{1} \ldots \sigma_{p}} & \delta_{\beta_{1}}^{\theta_{1}} \ldots \delta_{\beta_{q}}^{\theta_{q}} \delta_{\gamma_{1}}^{\sigma_{1}} \ldots \delta_{\gamma_{q}}^{\sigma_{p}}
\end{array}\right)
$$

where $\widetilde{A}(\widetilde{A})^{-1}=\left(\widetilde{A}_{B}^{A}\right)\left(\widetilde{A}_{C}^{B}\right)^{-1}=\delta_{C}^{A}=\widetilde{I}$, where $A=(a, \alpha, \bar{\alpha}), B=(b, \beta, \bar{\beta}), C=(c, \theta, \bar{\theta})$.

Proof. In fact, from (16) and (17), we easily see that

$$
\begin{aligned}
\widetilde{A}(\widetilde{A})^{-1} & =\left(\widetilde{A}_{B}^{A}\right)\left(\widetilde{A}_{C}^{B}\right)^{-1}=\left(\begin{array}{ccc}
\delta_{b}^{a} & \partial_{\beta} x^{a} & 0 \\
0 & \delta_{\beta}^{\alpha} & 0 \\
0 & \partial_{\beta} \xi_{\alpha_{1} \ldots \alpha_{q}}^{\sigma_{1} \ldots \sigma_{p}} & \delta_{\alpha_{1}}^{\beta_{1}} \ldots \delta_{\alpha_{q}}^{\beta_{q}} \delta_{\gamma_{1}}^{\sigma_{1}} \ldots \delta_{\gamma_{p}}^{\sigma_{p}}
\end{array}\right)\left(\begin{array}{ccc}
\delta_{c}^{b} & -\partial_{\theta} x^{b} & 0 \\
0 & \delta_{\theta}^{\beta} & 0 \\
0 & -\partial_{\theta} \xi_{\beta_{1} \ldots \beta_{q}}^{\sigma_{1} \ldots \sigma_{p}} & \delta_{\beta_{1}}^{\theta_{1}} \ldots \delta_{\beta_{q}}^{\theta_{q}} \delta_{\gamma_{1}}^{\sigma_{1}} \ldots \delta_{\gamma_{q}}^{\sigma_{p}}
\end{array}\right) \\
& =\left(\begin{array}{ccc}
\delta_{c}^{a} & -\partial_{\theta} x^{a}+\partial_{\theta} x^{a} & 0 \\
0 & \delta_{\theta}^{\alpha} & 0 \\
0 & \partial_{\theta} \xi_{\alpha_{1} \ldots \alpha_{q}}^{\sigma_{1} \ldots \sigma_{p}}-\partial_{\theta} \xi_{\alpha_{1} \ldots \alpha_{q}}^{\sigma_{1} \ldots \sigma_{p}} & \delta_{\alpha_{1}}^{\theta_{1} \ldots} \delta_{\alpha_{q}}^{\theta_{q}}
\end{array}\right)=\left(\begin{array}{ccc}
\delta_{c}^{a} & 0 & 0 \\
0 & \delta_{\theta}^{\alpha} & 0 \\
0 & 0 & \delta_{\alpha}^{\theta}
\end{array}\right)=\delta_{C}^{A}=\widetilde{I} .
\end{aligned}
$$

Let $A \in \mathfrak{I}_{q}^{p}\left(B_{m}\right)$. If we take account of (5) and (16), we can easily prove that ${ }^{v v} A^{\prime}=\widetilde{A}\left({ }^{v v} A\right)$, where ${ }^{v v} A \in \mathfrak{I}_{0}^{1}\left(t_{q}^{p}\left(B_{m}\right)\right)$ is a vector field defined by

$$
{ }^{v v} A=\left(\begin{array}{c}
{ }^{v v} A^{a} \\
{ }^{v v} A^{\alpha} \\
{ }^{v v} A^{\bar{\alpha}}
\end{array}\right)=\left(\begin{array}{l}
0 \\
0 \\
A_{\beta_{1} \ldots \beta_{q}}^{\alpha_{1} \ldots \alpha_{p}}
\end{array}\right),
$$

with respect to the adapted frame $\left\{B_{(b)}, C_{(\beta)}, C_{(\bar{\beta})}\right\}$ of $\beta_{\xi}\left(M_{n}\right)$.

Taking account of (9), (10) and (16), we see that the complete $\operatorname{lift}{ }^{c c} X$ and horizontal $\operatorname{lift}{ }^{H H} X$ have respectively 
components

$$
{ }^{c c} \widetilde{X}:\left(\begin{array}{l}
\widetilde{X}^{b} \\
X^{\beta} \\
-L_{X} \xi_{\beta_{1} \ldots \beta_{q}}^{\alpha_{1} \ldots \alpha_{p}}
\end{array}\right),{ }^{H H} \widetilde{X}:\left(\begin{array}{l}
\widetilde{X}^{b} \\
X^{\beta} \\
\left(\nabla_{X} \xi\right)_{\beta_{1} \ldots \beta_{q}}^{\alpha_{1} \ldots \alpha_{p}}
\end{array}\right)
$$

with respect to the adapted frame $\left\{B_{(b)}, C_{(\beta)}, C_{(\bar{\beta})}\right\}$ of $\beta_{\xi}\left(M_{n}\right)$, where ${ }^{c c} \widetilde{X}^{\prime}=\widetilde{A}\left({ }^{c c} \widetilde{X}\right)$ and ${ }^{H H} \widetilde{X}^{\prime}=\widetilde{A}\left({ }^{H H} \widetilde{X}\right)$.

We now, from equations (7), (8) and (16) see that $\gamma \varphi$ and $\widetilde{\gamma} \varphi$ have respectively components

$$
\gamma \varphi=(\gamma \varphi)^{I}=\left(\begin{array}{l}
0 \\
0 \\
\sum_{\lambda=1}^{p} \xi_{\beta_{1} \ldots \beta_{q}}^{\alpha_{1} \ldots \alpha_{p}}
\end{array}\right), \widetilde{\gamma} \varphi=(\widetilde{\gamma} \varphi)^{I}=\left(\begin{array}{l}
0 \\
0 \\
\sum_{\mu=1}^{q} \xi_{\beta_{1} \ldots \varepsilon \ldots \beta_{q}}^{\alpha_{1} \ldots \alpha_{p}} \varphi_{\beta_{\mu}}^{\varepsilon}
\end{array}\right)
$$

with respect to the adapted frame $\left\{B_{(b)}, C_{(\beta)}, C_{(\bar{\beta})}\right\}$ of $\beta_{\xi}\left(M_{n}\right)$.

Let $S \in \mathfrak{I}_{2}^{1}\left(M_{n}\right)$ now. If we take account of (16), we see that $(\gamma S)^{\prime}=\widetilde{A}(\gamma S) \cdot \gamma S$ is given by

$$
\gamma S=(\gamma S)_{J}^{I}=\left(\begin{array}{lcr}
0 & 0 & 0 \\
0 & 0 & 0 \\
0 \sum_{\lambda=1}^{p} S_{\beta_{\varepsilon}}^{\beta_{\lambda} \xi_{\alpha_{1} \ldots \alpha_{q}}^{\beta_{1} \ldots \ldots \ldots \beta_{p}}} & 0
\end{array}\right)
$$

with respect to the adapted frame $\left\{B_{(b)}, C_{(\beta)}, C_{(\bar{\beta})}\right\}$, where $S_{\beta_{\varepsilon}}^{\beta_{\lambda}}$ are local componenets of $S$ on $B_{m}$.

$B X, C X$ and $E \xi$ also have components:

$$
B X=\left(\begin{array}{l}
X^{\alpha} \\
0 \\
0
\end{array}\right), C X=\left(\begin{array}{l}
0 \\
X^{\alpha} \\
0
\end{array}\right), E \xi=\left(\begin{array}{l}
0 \\
0 \\
\xi_{\beta_{1} \ldots \beta_{q}}^{\alpha_{1} \ldots \alpha_{p}}
\end{array}\right)
$$

respectively, with respect to the adapted frame $\left\{B_{(b)}, C_{(\beta)}, C_{(\bar{\beta})}\right\}$ of the cross-section $\beta_{\xi}\left(M_{n}\right)$ determined by a tensor field $\xi$ of type $(p, q)$ in $M_{n}$.

\section{Acknowledgment}

The authors would like to thanks the referees for useful and improving comments.

\section{Competing interests}

The authors declare that they have no competing interests.

\section{Authors' contributions}

All authors have contributed to all parts of the article. All authors read and approved the final manuscript. 


\section{References}

[1] T.V. Duc, Structure presque-transverse. J. Diff . Geom., 14(1979), No:2, 215-219.

[2] C.J. Isham, "Modern differential geometry for physicists", World Scientific, 1999.

[3] H. Fattaev, The Lifts of Vector Fields to the Semitensor Bundle of the Type (2, 0), Journal of Qafqaz University, 25 (2009), no. 1, 136-140.

[4] A. Gezer and A. A. Salimov, Almost complex structures on the tensor bundles, Arab. J. Sci. Eng. Sect. A Sci. 33 (2008), no. 2, 283-296.

[5] D. Husemoller, Fibre Bundles. Springer, New York, 1994.

[6] V. Ivancevic and T. Ivancevic , Applied Differential Geometry, A Modern Introduction, World Scientific, Singapore, 2007.

[7] H.B. Lawson and M.L. Michelsohn, Spin Geometry. Princeton University Press., Princeton, 1989.

[8] A.J. Ledger and K. Yano, Almost complex structure on tensor bundles, J. Dif. Geom. 1 (1967), 355-368.

[9] A. Salimov, Tensor Operators and their Applications. Nova Science Publ., New York, 2013.

[10] A. A. Salimov and E. Kadığlu, Lifts of derivations to the semitangent bundle, Turk J. Math. 24 (2000), 259-266.

[11] N. Steenrod, The Topology of Fibre Bundles. Princeton University Press., Princeton, 1951.

[12] V. V. Vishnevskii, Integrable affinor structures and their plural interpretations. Geometry, 7.J. Math. Sci. (New York) 108 (2002), no. 2, 151-187.

[13] G. Walschap, Metric Structures in Differential Geometry, Graduate Texts in Mathematics, Springer-Verlag, New York, 2004.

[14] F. Yıldırım, On a special class of semi-cotangent bundle, Proceedings of the Institute of Mathematics and Mechanics, (ANAS) 41 (2015), no. 1, 25-38.

[15] F. Yıldırım and A. Salimov, Semi-cotangent bundle and problems of lifts, Turk J. Math, (2014), 38, 325-339. 